\title{
LEARNING AUTOMATA BASED METHOD FOR GRID COMPUTING RESOURCE VALUATION WITH RESOURCE SUITABILITY CRITERIA
}

\author{
Ali Sarhadi \\ Department of Computer Engineering, \\ Malayer Branch, Islamic Azad University Malayer, Iran \\ Sarhadi_alieiau-malayer.ac.ir
}

\begin{abstract}
In economic Grid environments, the producers (resource owners) and consumers (resource users) have different goals, objectives, strategies, and supply-and-demand patterns. Mechanism based on economic models is an effective approach to solve the problem of grid resources management. Grid resource valuation and allocation is one of the fundamental problems in grid resource management. The essence of this problem is how to allocate and valuation resources for achieving the goal of a highly efficient utilization of resources in response to current resource valuations. Pricing policies are based on the demand from the users and the supply of resources is the main driver in the competitive, economic market model. In this paper, we present a new method of resource allocation and valuation based on the learning automata algorithms in order to maximize the benefit for both grid providers and grid users. We formulate the problem as an environment that learning automata's allocate best resource based on its complete time for proffered application. After allocate of resource, valuation of it based on its complete time is done. With this method the valuation of resource is based on their suitability for jobs execution. Using computer simulations, it is shown that the proposed methodology have higher performance in comparing with existing methods.
\end{abstract}

\section{KEYWORDS}

Economic grid; resource valuation; learning automata; grid computing

\section{INTRODUCTION}

Grid computing is an emerging technology that enables users to share a large number of computing resources distributed over a network. The dynamic, federating nature of Grid policy environments is dominated by virtual organizations (VOs) which associate heterogeneous users and resource providers[1][2].

Users have resource-consuming activities, or jobs, that must be mapped to specific resource providers through a resource allocation mechanism. The resource allocation mechanism may choose among alternate mappings in order to optimize some utility metric, within the bounds permitted by the VO policy environment. 
It is envisioned that deployment of Grid technology will grow from its current modest scale to eventually overlay the global Web. It is not known how large individual VOs will be, but it is reasonable to imagine resource sharing among populations with tens of thousands of users and thousands of resources. Hence, allocation mechanisms need to be highly scalable and robust to localized failures in resources and communication paths. From the perspective of a single VO, the dynamic policy environment can be viewed as the dynamic arrival and departure of users and resources (occurring at a higher rate than users and resources actually associate with and disassociate from the global Grid infrastructure). Scalable Grid allocation mechanisms need to focus on the VO policy environment rather than physical locations. Recently economic grid has been growing. The introduction of economics-based models in Grid resource management involves the definition of a computational market, in which Grid users and providers interact. Resource valuation is one of the fundamental aspects of economic grid computing [3]. An important part of the interaction is the establishment of valuations levels. Valuations levels should reflect the participants' valuations of the goods traded. A valuations scheme that determines these price levels throughout the Grid's operational time frame has to be devised. Ideally, the valuations scheme should be dynamic, depending on demand, supply, value, and general wealth levels.

When there is a period of high demand, valuations should rise. In addition to a valuation scheme, agreements have to be made on the type and granularity of the goods that are traded in the market. Furthermore, an agreement has to be made on the currency used. Indeed, according to [5], virtual economies are worthless unless the currency used can somehow be converted to real world money. While this is certainly our long term goal, we have currently chosen not to make this link, as it would introduce its own set of problems such as exchange rates, security, management systems, and so on. It is clear that framing the resource allocation problem in economic terms is attractive for several reasons [4]: resource usage is not free, the dynamics of Grid performance response are hard to model, and it provides an incentive for resource owners to bring their resources to the Grid. For the above reasons, resource management based on economic principles will play an important part in the next generation of Grid related challenges, which will be dominated by economical aspects instead of technical ones [5]. One of the main challenges in economic grid is grid resource valuation. Many method for resource valuation in grid computing environment are represented such as Fixed Price Model‘Posted Price Model‘Bargaining Model‘Tendering/Contract-net Model‘Auction Model‘Cooperative Bartering Model‘ Monopoly Oligopoly [5][6][7][8][9][11]. But none of these methods do not focus on valuation of computational resource based on their suitability and capability in executing of applications. In this paper we try solve this problem by representing a new model of computing resource valuation in economic grid using learning automata [16]. The proposed approach uses learning automata for resource allocation with shortest time in executing of proffered application and then resource valuation based on its total time for executing of proffered application estimate.

\section{RELATED WORK}

Cost-based resource management and market-oriented models have proven to be useful for resource management in the context of cluster computing [2]. Furthermore, economic models for resource sharing have been applied to agent systems [10,11], telecommunication networks [12], databases [10] and data mining [13], optimization of business value in utility data centers [7], and more recently to Grid computing [3]. An overview can be found in [13].

According to the classification in [6], our work uses a state-based, non-pre-emptive strategy. State-based means that the allocations are based on a current snapshot of the system state as opposed to model-based, where the allocations are based on a predictive model. Non-pre-emptive means that tasks are assigned to hosts once and stay there, as opposed to pre-emptive strategies 
where tasks are allowed to migrate between hosts. The use of a commodity market model for obtaining global equilibrium prices for resources in a Grid context has already been proposed in $[19,20]$. We take this approach further and extend it to allow for trading and pricing of substitutable goods. This more closely models Grid markets, where multiple resources are available to process the jobs, but with varying performance characteristics. We also introduce significant improvements to the optimization algorithms used to compute the equilibrium prices.

\section{LEARNING AUTOMATA}

Learning Automata [17] are adaptive decision-making devices operating on unknown random environments. A Learning Automaton has a finite set of actions and each action has a certain probability (unknown to the automaton) of getting rewarded by the environment of the automaton that Equation (1) has been shown desire response. The aim is to learn to choose the optimal action through repeated interaction on the system. If the learning algorithm is chosen properly, then the iterative process of interacting on the environment can be made to result in selection of the optimal action. Figure 1 illustrates how a stochastic automaton works in feedback connection with a random environment. Learning Automata can be classified into two main families: fixed structure learning automata and variable structure learning automata (VSLA) [16]. In the following, the variable structure learning automata which will be used in this paper is described.

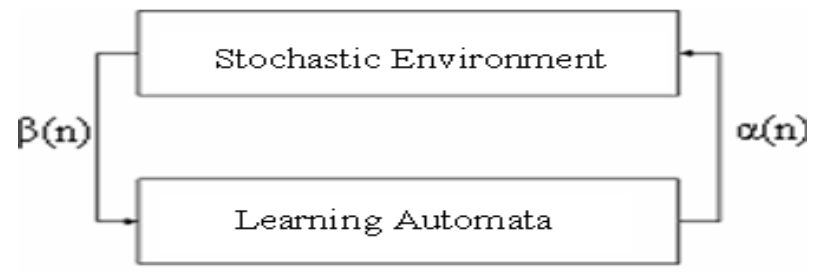

Figure 1. The interaction between learning automata and environment

A VSLA is a quintuple $<\alpha, \beta, \mathrm{p}, \mathrm{T}(\alpha, \beta, \mathrm{p})>$, where $\alpha, \beta, \mathrm{p}$ are an action set with s actions, an environment response set and the probability set $\mathrm{p}$ containing s probabilities, each being the probability of performing every action in the current internal automaton state, respectively. If the response of the environment takes binary values learning automata model is P-model and if it takes finite output set with more than two elements that take values in the interval $[0,1]$, such a model is referred to as Q-model, and when the output of the environment is a continuous variable in the interval $[0,1]$, it is refer to as S-model. The function of $\mathrm{T}$ is the reinforcement algorithm, which modifies the action probability vector $\mathrm{p}$ with respect to the performed action and received response. Assume $\beta \in[0,1]$. A general linear schema for updating action probabilities can be represented as follows. Let action i be performed then:

A) Desire response

$$
\begin{aligned}
& p_{i}(n+1)=p_{i}(n)+a\left[1-p_{i}(n)\right] \\
& p_{j}(n+1)=(1-a) p_{j}(n) \quad \forall j j \neq i
\end{aligned}
$$

B) Undesired response

$$
\begin{aligned}
& p_{i}(n+1)=(1-b) p_{i}(n) \\
& p_{j}(n+1)=\frac{b}{r-1}+(1-b) p_{j}(n) \quad \forall j \quad j \neq i
\end{aligned}
$$

Where $a$ and $b$ are reward and penalty parameters. When $a=b$, the automaton is called $L_{R P}$. If $b=0$ the automaton is called $L_{R I}$ and if $0<b<<a<1$, the automaton is called $L_{R \varepsilon p}$. For more Information about learning automata the reader may refer to [17]. 
International Journal of Grid Computing \& Applications (IJGCA) Vol.2, No.4, December 2011

\section{GRID RESOUCE ALLOCATION BASED ON LEARNING AUTOMATA}

A number of approaches have been proposed which use economic models to address resource usage and incentives in a Grid [7]. Particularly, a well-designed market-based resource allocation mechanism provides incentives for participation by ensuring that all the actors in the system maximize their utility and do not have incentives to deviate from the designed protocol . In this paper we used of new mechanism for grid resource allocation based on learning automata. Then selected resource based on its competence in job execution will be priced. In this section we briefly introduced proposed method.

We need to describe how the learning automata's select resources. As the name learning suggests, automata's use their past experience to choose between the resources. In pervious section we introduce learning automata. A Learning Automaton has a finite set of actions and each action has a certain probability (unknown to the automaton) of getting rewarded by the environment of the automaton in our model for each possible action (i.e., selecting a specific resource) the LA keeps environment response that indicates the efficiency of that resource in the past and based on this response give reward or penalty to this action. Qualifications for grant of reward or penalty will be introduced in algorithm. After each selection, the LA gets a response from environment (containing the price of resource and completion time), calculates the complete time, and translates it into a reward $\mathrm{R}$ or penalty $\mathrm{P}$ for resource number i. For each new user, LA's chooses a resource with the highest reward. A Resource with minimum cost that execute users job in specified deadline has a higher reward and will be selected.

\section{PRICING POLICY}

These define the prices that resource owners would like to charge users. The resource owners may follow various policies to maximize their profit and resource utilization and the price they charge may vary with time and one user to another user. The pricing can also be driven by demand and supply like in the real market environment. That is, in this commodity market model, pricing is essentially determined by objective functions of service providers and users[14]. The pricing policy can also be based on auction. In this auction based economic model, pricing is driven by how much users value the service and the highest bidder wins the access to Grid services. Unfortunately this method and other methods for grid computing resource pricing are not based on resource competence. Often existing criteria for the grid resource pricing is unrealistic. So in this paper proposed model is based on resource competence by using of learning automata at the core of resource allocation.

\section{PROPOSED RESOURCE PRICING ALgORITHM}

We have tried in this method with using a set of learning automata at the core of resource allocation that mentioned in a above section and illustrated in the fig. 3. Incoming task is to allocate the best resource with time optimization criteria. When all of incoming tasks choose their resource, pricing of them is beginning. Resource discovery and pricing method illustrated in fig.3. 


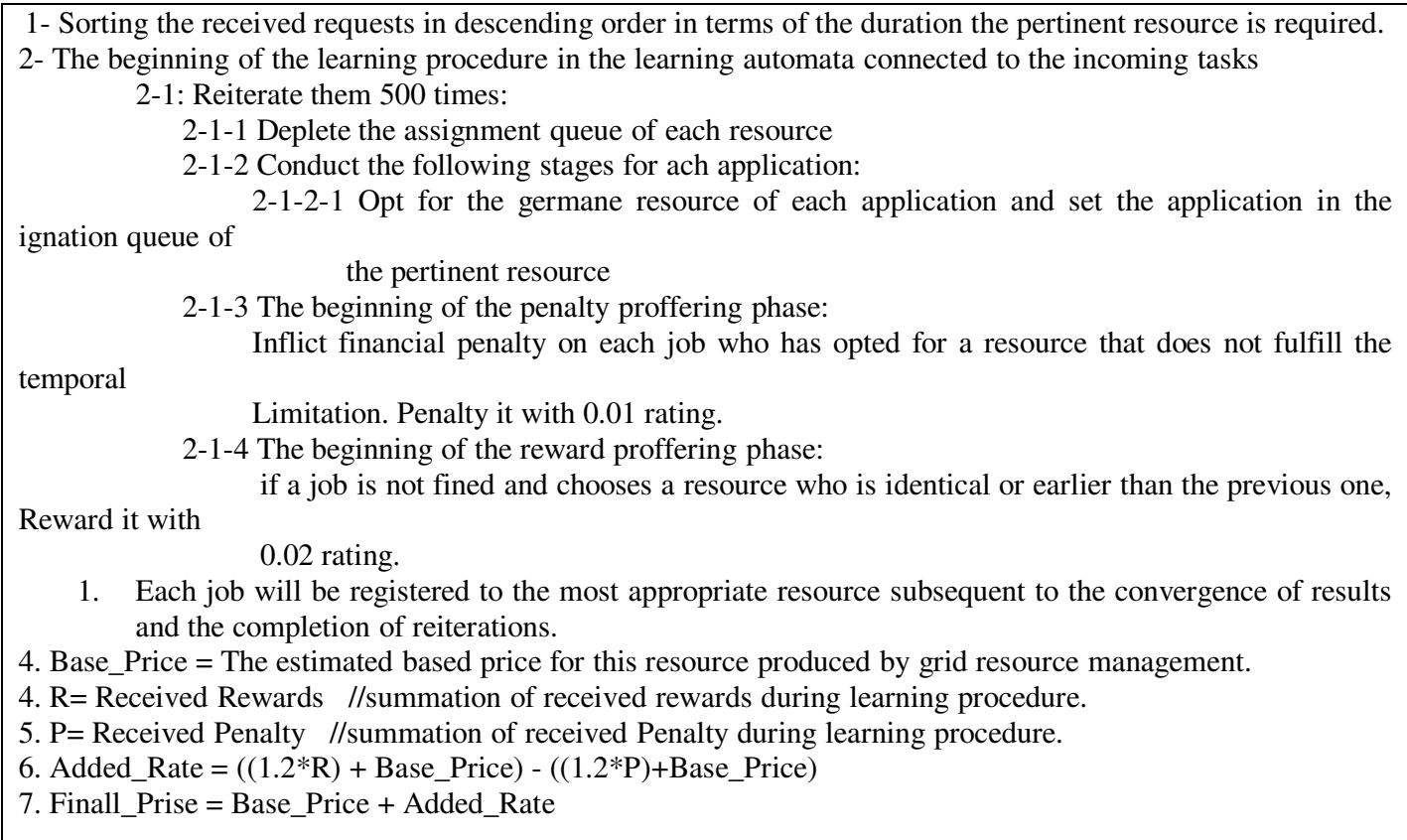

Figure 2. proffered algorithm

\subsection{CRITERIA FOR SELECTING REWARD AND PENALTY BY LEARNING AUTOMATA}

Environment response for learning automata associated to job based on ideal conditions in a time optimization criteria will be complete. For this propose environment uses of blew tow point for give reward or penalty to selected action by learning automata.

1- Run time of transferred application to busiest resources shouldn't have many differences run time with other resource.

2- Each work must be assigned to resource that can run it earlier from other resources

Thus the environment by using the first points will give penalty to selected action and with second point give reward to selected action by learning automata.

\section{EXPERIMENTS}

The goal of this experiment is to compare the performance of the learning automata based resource allocation and pricing algorithm to other conventional resource allocation approach such as $\mathrm{p} 2 \mathrm{p}$ model [26], which no pricing is used, and the incoming task queries are matched with the next available resource offer which meets the task's constraints. We simulate grid environment to evaluate experimentally the learning automata-based resource allocation and pricing algorithm by means of gridsim[18].

The most suitable parameters for evaluation of this algorithm have been propounded with regard to the effectuated securitizations. The error rate parameter expenditure improvement algorithms are regulated with regard to each selection for instance in failure cases of allocation, or in cases demanding re-allocation. Another parameter is the wait time that defines as duration of times 
before allocation of resource to a task and total time is complete time of a task. And finally efficiency of proffered algorithm in compared of conventional method will be shown. Reported results, is for average 20 times of simulation. Grid environment including a number of resources and users. Grid resource is high heterogeneous.

We neglect the network topology and the communication costs associated with it. Instead, we assume that each of the users can submit jobs to any of the resources. However, it is still adequate for certain situations. This experiment is to study allocation and pricing efficiency of learning automata-based resource allocation and pricing algorithm in terms of conventional resource discovery efficiency. In this experiment, we choose respectively 100, 200, 300, and 400 resource domains to compare resource allocation efficiency of the learning automata-based resource allocation and pricing algorithm to the conventional approach under various the numbers of resource domains. The experiment results are shown in blew for resource allocation efficiency.

From the results in Fig. 3, when the number of resource domains is low, the difference in resource allocation efficiency between the two allocation methods is small, and both can achieve good allocation efficiency, so using conventional approach might be sufficient. When the number of resource domains increases, resource allocation efficiency of both methods will decrease. However, the resource allocation efficiency of approach might be sufficient. When the number of resource domains increases, resource allocation efficiency of both methods will decrease. However, the resource allocation efficiency of learning automata-based resource allocation and pricing algorithm decreases slower than that of the conventional approach. In other words, for a large number of resource domains, the conventional approach will not match the performance of the learning automata-based resource allocation and pricing algorithm. From above performance comparisons, we can see that the resource allocation efficiency of the learning automata-based resource allocation and pricing method is effective improved.

As the Fig. 4 show Completion time of allocated job to the resourcer allocation by the proffered algorithm is less than to completion time of the same job allocated to other resources. But as shown in Fig. 3 the error rate in applying the proffered algorithm because of repeated learning automata to reach the desired resource is more. Fig. 5 shows the relation between completion time and resource price based on proffered algorithm. As you can see with decrease in completion time resource price will increase.

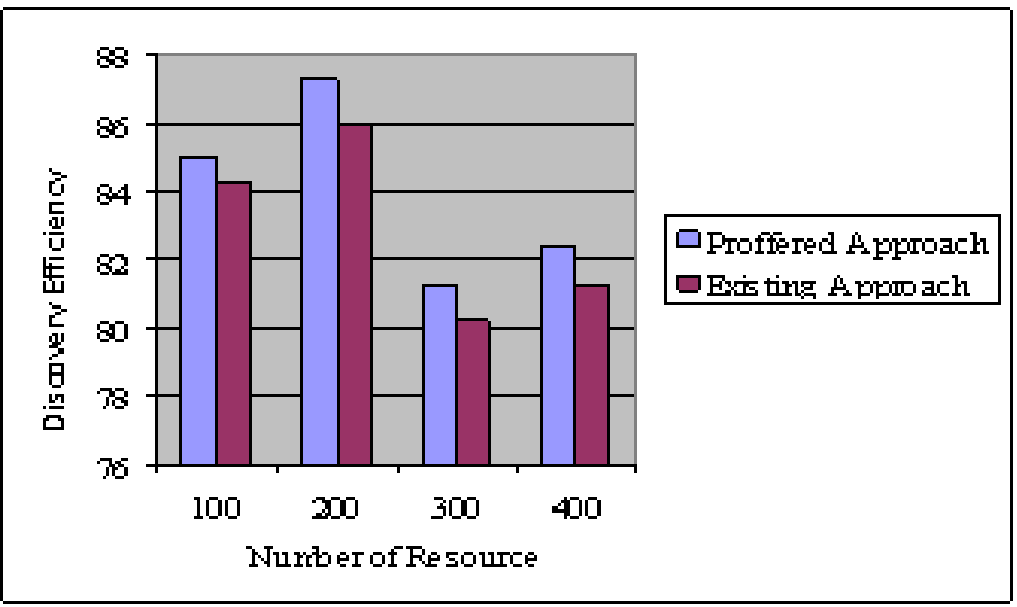

Figure 3 : Resource Discovery Efficiency 


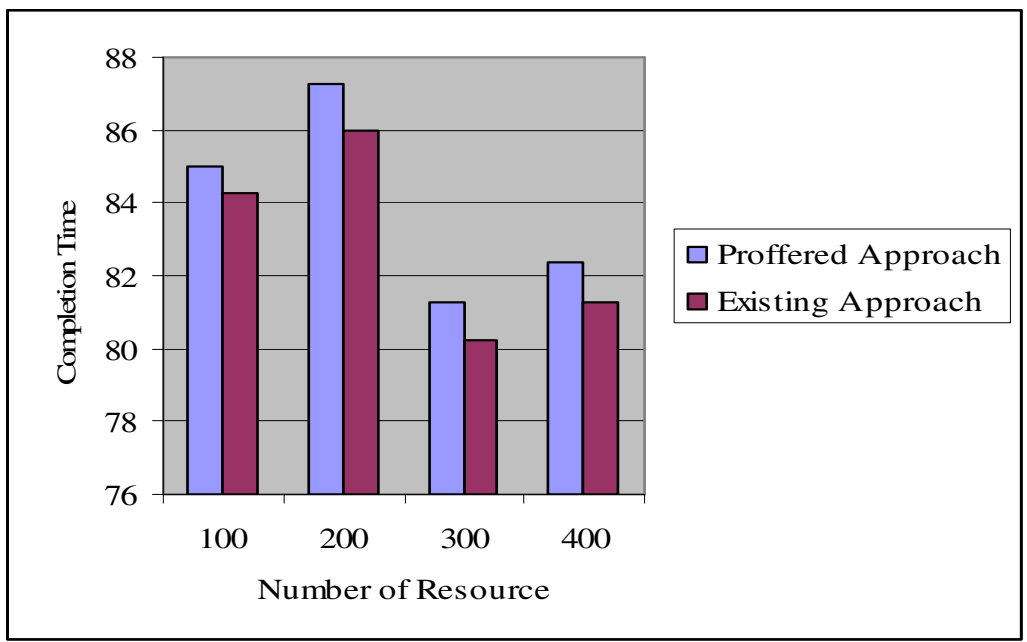

Figure 4 : Completion Time

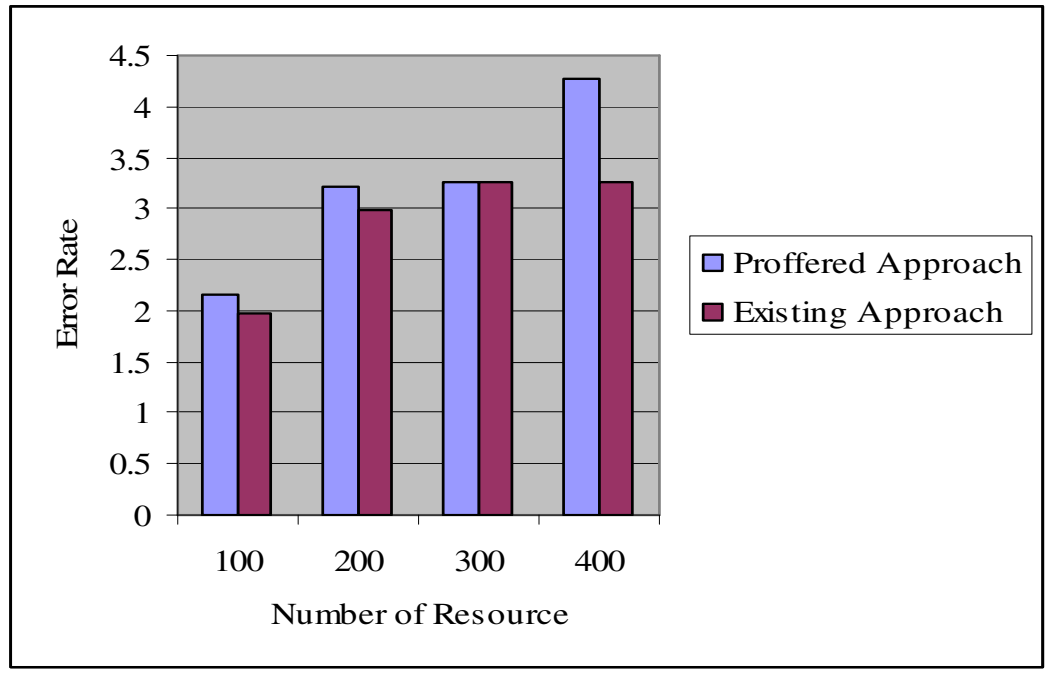

Figure 5 : Error Rate

\section{Conclusions}

Resource allocation and pricing based on learning automata is an effective approach to manage resources in economic grid computing environment. In this paper, we proffer a resource allocation and pricing method based on learning automata model in detail, which consists of set of learning automata associated to user's job to select best resource for executing applications and finally based on obtained reward and penalty, resource price will be evaluated. Efficiency of proffered algorithm based an Error rate and Completion time parameters were shown.

\section{ACKNOWLEDGEMENT}

This work is partially supported by the Islamic Azad University-Malayer branch. 
International Journal of Grid Computing \& Applications (IJGCA) Vol.2, No.4, December 2011

\section{REFERENCE}

[1] Foster, I., Kesselman, C.(eds.).: The Grid: Blueprint for a New Computing Infrastructure. Morgan Kaufmann (2006)

[2] Foster, I., Kesselman, C., Tuecke S.: The Anatomy of the Grid: Enabling Scalable Virtual Organizations. International Journal of High Performance Computing Application, 15(3) (2008) 200222

[3] Foster, I., Kesselman, C., Nick, J., Tuecke, S.: The Physiology of the Grid: An Open Grid Services Architecture for Distributed Systems Integration.

[4] Buyya, R., Abramson, D., Giddy, J.: A Case for Economy Grid Architecture for Service Oriented Grid Computing. Proceedings of International Parallel and Distributed Processing Symposium: Heterogeneous Computing Workshop (HCW 2005), San Francisco, USA.

[5] R. Wolski, J. Plank, J. Brevik, and T. Bryan: Analyzing Market-based Resource Allocation Strategies for the Computational Grid. International Journal of High performanceComputing Applications, Sage Publications, 2007,Vol 15(3), 258-281

[6] Subramoniam, K., Maheswaran, M., Toulouse, M.: Towards a Micro-Economic Model for Resource Allocation in Grid Computing System. Proceedings of the 2009 IEEE Canadian Conference on Electrical and Computer Engineering, 2008, 782-785

[7] Buyya, R.: Economic-based Distributed Resource Management and Scheduling for Grid Computing. $\mathrm{PhD}$ Thesis, Monash University, Melbourne, Australia, April 12,2002. Online at http://www.buyya.com/thesis/

[8] B.N. Chun, D.E. Culler, User-centric performance analysis of market based cluster batch schedulers, in: Procs of the 2nd IEEE/ACM Int'l Symposium on Cluster Computing and the Grid, IEEE Computer Society, Washington, DC, USA, 2007, pp. 22-30.

[9] J. Sherwani, N. Ali, N. Lotia, Z. Hayat, R. Buyya, Libra: A computational economy-based job scheduling system for clusters, Software Practice and Experience 34 (6) (2009) 573-590.

[10] J. Bredin, D. Kotz, D. Rus, R.T. Maheswaran, C, . Imer, T. Bas,ar, A market based model for resource allocation in agent systems, in: Coordination of Internet Agents: Models, Technologies, and Applications, Springer-Verlag, London, UK, 2010, pp. 426-442.

[11] J. Bredin, R.T. Maheswaran, C . Imer, T. Bas sar, D. Kotz, D. Rus, Computational markets to regulate mobile-agent systems, Autonomous Agents and Multi-Agent Systems 6 (3) (2008) 235-263.

[12] A. Byde, M. Salle, C. Bartolini, Market-based resource allocation for utility data centers, Tech. Rep. HPL-2003-188, HP Labs, 2009.

[13] D. Abramson, R. Buyya, J. Giddy, A computational economy for Grid computing and its implementation in the nimrod-g resource broker, Future Generation Computer Systems 18 (8) (2009) 1061-1074.

[14] K. Vanmechelen, G. Stuer, J. Broeckhove, Pricing substitutable Grid resources using commodity market models (Proceedings of the 3rd nternationalWorkshop on Grid Economics and Business Models),World Scientific, Singapore, 2006, pp. 103-112.

[15] Chuliang Weng, Xinda Lu, Qianni Deng: A Distributed Approach for Resource Pricing in Grid Environments. In: Proceedings of the Second International Workshop on Grid and Cooperative Computing (GCC 2003), Shanghai, China, December 7-10, 2005, LNCS, Vol.3033,(2005) 620 - 627. 
International Journal of Grid Computing \& Applications (IJGCA) Vol.2, No.4, December 2011

[16] K. Narendra and M. A. L. Thathachar, Learning Automata: An Introduction, Prentice Hall, Englewood Cliffs, New Jersey, 2004.

[17] K. Najim and A. S. Poznyak, Learning Automata: Theory and Application, Elsevier Science Ltd., Tarrytown, NY, 2005.

[18] R. Buyya and M. Murshed, GridSim: A Toolkit for the Modeling and Simulation of Distributed Resource Management and Scheduling for Grid Computing, Technical Report, Monash University, Nov. 2008. To appear in the Journal of Concurrency and Computation: Practice and Experience (CCPE), 1-32pp, Wiley Press, May 2008. 